\title{
Moving Crack detection based on Improved VIBE and Multiple Filtering in Image Processing Techniques
}

\author{
Ahmed Mahgoub Ahmed Talab ${ }^{1,2}$, Zhangcan Huang ${ }^{1,{ }^{*}}$, FangXi $^{1}$,LiuHaiming ${ }^{1}$. \\ 1. School of Sciences, Wuhan University of Technology, Wuhan; 43007,China. \\ 2. College of Engineering, Elimam ELMahdi University, Kosti, Sudan. \\ ahmedtalab10@yahoo.com; huangzc@whut.edu.cn; fangxi@whut.edu.cn ; \\ 1golden@163.com.
}

\begin{abstract}
Background subtraction is important process in many automatic video content analysis applications. extracting foreground moving objects from video sequences is a crucial step and also a hot topic in computer vision and image processing. This paper presents a new approach in image processing for detection crack in video. This method involves two main steps: First, we use based on Visual Background Extractor (Vibe) to detection moving crack in video. Second steps: Using a suitable threshold in a binary image and classifies all pixels two groups' background and foreground. And use filter area and changes the area if less than the specific number to back; and using filtering to elimination of residual noise. This paper describes a method for detection crack in video we use Image Processing Techniques. The advantage of this method is clearly and accurate detection of cracks in video. Experimental work shows that our method is improved relatively to the other widely used techniques.
\end{abstract}

Keywords: moving object detection; VIBE Filtering Image; moving Crack detection

\section{Introduction}

Background subtraction is one of the main and widely used tools in automatic video content analysis, particularly in video surveillance[1-3]. However numerous acceptable techniques have been aimed so far for the background extraction, but there is still need to produce more effective methods in terms of adaptability to various environments, noise resilience, and computation efficiency[1]. Over the past years, several, methods for background subtraction techniques have been proposed[4, 5]. Similarly, moving object's detection for a static camera has been extensively studied for many years [6]. Moving objects detection is very a crucial step in many vision applications with the purpose of subtracting interesting target area and locating the moving objects from image sequences. It is wide used in vision systems such as traffic control, video surveillance of objects, video surveillance of unattended outdoor environments, activity recognition, object tracking and behavior understanding. Accurate moving object detection is necessity for the robustness of intelligent video surveillance systems[3]. Background subtraction and temporal differencing are two popular approaches to segment moving objects in an image succession under a stationary camera. Background subtraction detects moving objects in an image by assessing the difference of pixel features of the current scene image against the reference background image, such as the Gaussian mixture model (GMM) [7-9].GMM is a widely used approach because of its self learning ability and its robustness to variations in lighting. But, it still has some shortcomings. One problem is that it does not explicitly model the spatial dependences of neighboring background pixels' colors. hence, some false positive pixels will be produced in highly dynamic scenes where dynamic texture does not repeat exactly[3]. Olivier Barnich and Marc Van Droogenbroeck proposed 
Vibe algorithm [1, 2].The algorithm adopt neighboring pixels to create the background model, by comparison the background model and the current input pixel values to detect targets, the method also gives three steps to update the area to adapt to changes in the environment. It utilizes randomly selected old samples to approximate the color distribution of the background. The feature of the randomization is that it avoids replacement of the oldest samples. Still, when the background have similar color with foreground, it will result in missing the foreground point and thus cause lack of accurate contour for post motion analysis like recognition. When the foreground object through these areas, the detection precision will drop; at the same time, goes up the high rate of false detection algorithm because of the ghosting region [3].

In this paper, in order to overcome the shortcoming of origin VIBE and supply accurate silhouette with good spatial and temporal consistency, we present a novel moving object detection method based on improved VIBE and Multiple Filtering in image processing techniques from detection crack in video. Firstly, execute moving object detection for the current frame based on VIBE method to extract the background and foreground information; Secondly, Using multiple filtering to detection clear crack in video ; and calculate the all foreground pixel less than specific number to background ; And use filter of area to elimination of residual noise. Experimental results on video demonstrate the efficiency of our proposed method. The paper is organized as follows: Section 2 covers and outlines the related algorithms to the proposed method and explains and outline the proposed method section 3 design the specimens of this work. Section 4 shows experimental results. Finally, section 5 presents this work conclusion.

\section{Algorithms in this Work}

\subsection{Outline of VIBE algorithm}

Olivier suggests the application of Visual Background Extractor (ViBe) as a general background subtraction method. The algorithm follows neighboring pixels to institute the background model by comparing the background model with the current pixel value. The execution of the algorithm is separated into the following steps[1-3].The first step is to format the single frame image from each pixel in the background model. Because in a single frame, there is no transitory information, it is thought that adjacent pixels contribute an identical transit distribution. This intends that the value of a pixel, and its neighbor pixel values in the spatial domain have a similar distribution. The correct size range of the neighborhood is selected to ensure that the background model admits an enough number of different samples, while take into account that as the neighborhood scope increases, the correlation between pixel values at different positions decreases. It is presumed that $\mathrm{T}=0$ refers to the first frame; $B G^{0}(x, y), N_{G}(x, y), P^{0}(x, y)$ is the pixel background model value, spatial neighborhood value, pixel value, then:

$$
B G^{0}(x, y)=\left\{P_{m}^{0}\left(x^{n}, y^{n}\right) \mid\left(x^{n}, y^{n}\right) \in N_{G}(x, y)\right\}
$$

Where $\left(x^{n}, y^{n}\right)$ in $N_{G}(x, y)$ is selected such as probability. $\mathrm{m}=1,2, \ldots, \mathrm{N}$ is the number of samples. Second step is a decision process to determine whether an input pixel belongs to the background or not, then to update the background pixel model. They declare by the pixel value $\mathrm{p}(\mathrm{x}, \mathrm{y})$ in a given Euclidean color space chosen by the pixel located at $(\mathrm{x}, \mathrm{y})$ in the image, and every background model is modeled by a collection of 20 background samples. when $\mathrm{T}=\mathrm{t}$, the background model of $(\mathrm{x}, \mathrm{y})$ is $B G^{t-1}(x, y)$, the pixel value is $P^{t}(x, y)$, the formula for determining the input frame image for foreground objects segmentation is as follows: 
$P^{t}(x, y)=\left\{\begin{array}{cl}\text { foreground } & \left|B G^{t-1}(x, y)-P^{t}(x, y)\right|>R \\ \text { background } & \left|B G^{t-1}(x, y)-P^{t}(x, y)\right|<R\end{array}\right.$

Where superscript $\mathrm{r}$ is chosen randomly; $\mathrm{R}$ is the fixed shold (which in color space is a spherical radius). when $P^{t}(x, y)$ is larger than or equal to a given background nominee \#min ; "the minimal cardinality"), we consider it is the corresponding background pixel, otherwise is the foreground. In Vibe algorithm, \#min is set to 2). It means that it is enough to determine at least two samples close enough to classify the pixel in the background. Because the being of a moving object in the first frame will introduce an artifact commonly called a ghost (regions of connected foreground points that do not correspond to any real object). Although using the later frames can update the background to remove the ghost, the process is a little slow. Because of the being of ghost, the precision of detection will be decreased. Otherwise, the detection result of Vibe method is sensitive to illumination changes, dynamic scenes, and cluttered background. The final step randomly update the background model with each new frame. Because of the strong statistical correlation between a pixel and its neighbor pixel, while a pixel is detected as the background pixel, its neighbor pixel is highly possible to be considered as the background pixel with high possibility. Accordingly, It is using the background pixels to update the background model of neighboring pixels. Through the random updating policy, we can consolidate the foreground objects which halt suddenly or stay long into the background model[3].

\subsection{The Proposed Method}

The object of this paper is proposing new approach at image processing techniques for detection crack in video. The proposed algorithm consists of main two steps:

First Step:

(1) Use the original video (RGB), and change the image to gray.

(2) Perform moving object detection for the current frame based on VIBE method to extract the background and foreground information.

(3) After get the background model, select one pixel model from all.

(4) Select one neighborhood from all, and detection foreground.

Second Step:

(5) Using a suitable threshold (T) in a binary image that classifies all pixels in two classifications background and foreground. According to this equation.

$$
I=\left\{\begin{array}{lr}
1 & \text { thr }>\mathrm{T} \\
0 & \text { otherwise }
\end{array}\right.
$$

(6) Use the function (bwlabed).

(7) Use the function (region props) to get the region area, and get the filter area.

(8) Change the small area than specific number to back. 


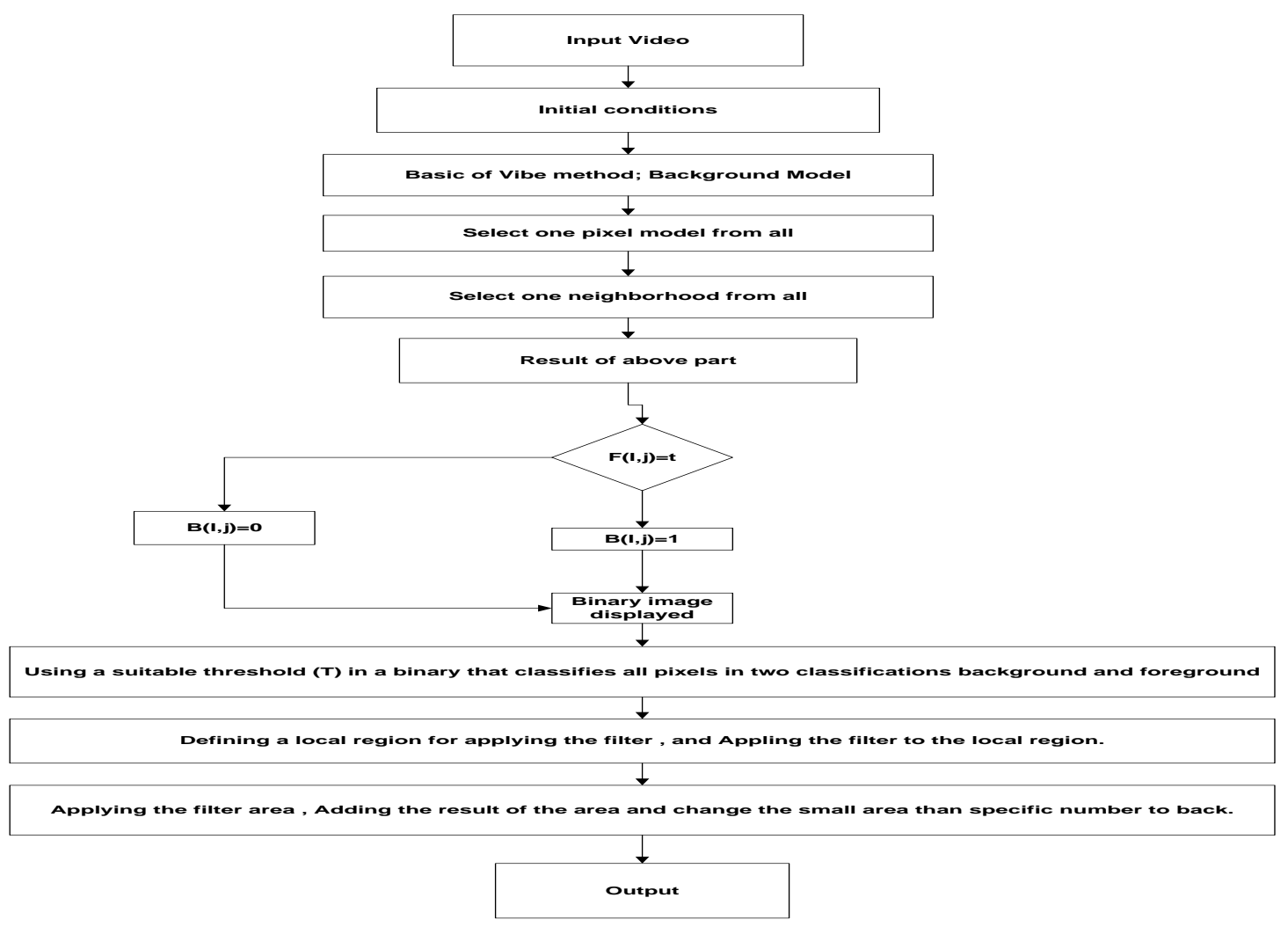

Figure.1. Flowchart for Proposed Method

\section{Experimental Program:}

\subsection{Raw Materials.}

Test using cement P.O 42.5 Huaxin cement ; the coal ash is I level which is from a thermal power plant ; quartz sand ,using an ordinary quartz sand, particle size were divided to 80-100 mesh, 40-70 mesh ; normal tap Water ; Grace ADVT using for reducing water super plasticizer. During the experiment we designed 3 kinds of the dosage of fly ash FA/C $=1.6,2.0$ and 2.4. The water-binder ratio is 0.31 , while the sandbinder ratio is 0.45 . All the material mixture ratios were shown specifically in table 1 .

Table1: Material Mixture Rations in Tensile Tests

\begin{tabular}{|c|c|c|c|c|c|}
\hline Cement & Fly ash & Sand & Water & $\begin{array}{l}\text { Super plasticizer } \\
\text { admixture }\end{array}$ & $\begin{array}{l}\text { Viscosity modifier } \\
\text { admixture }\end{array}$ \\
\hline 1 & 1.6 & 1.18 & 0.83 & 0.004 & 0.00075 \\
\hline 1 & 2 & 1.36 & 0.93 & 0.004 & 0.00075 \\
\hline 1 & 2.4 & 1.52 & 1.03 & 0.004 & 0.00075 \\
\hline
\end{tabular}

Note: The data shown in the table were the quality ratio.

\subsection{Preparation and Maintenance of Specimen.}

Using dumbbell - shaped for Uniaxial tensile tests specimens, Japanese standard reference, The size of the specimen is shown in figure 2 . The specimen as a preparation and process of materials test:

1- Mixed up the cement and fly ash with the quartz for 30s to ensure that all materials were mixed evenly . 
2-Stir : use $80 \%$ Super plasticizer and water it should keep continuous-stirring for at least 2 min until we could obtain the flow able foundation.

3 - Curing : demoulding after curing for $24 \mathrm{~h}$ at room temperature and then curing in the standard curing box. The temperature in the box was $20 \pm 0.5{ }^{\circ} \mathrm{C}$, humidity is about $97 \pm 1 \%$. Curing time is about 28 days.

\section{Experimental Test Method}

\subsection{Equipment}

For Uniaxial tensile test using Universal Testing Machine (Instron 5882), the Loading speed ratio was $0.4 \mathrm{~mm} / \mathrm{min}$ which was controlled under the displacement control mode.

\subsection{Test Method:}

Data collection, including capacity and strain. The test method referred to the Japanese standard. The strain data was measured by the 10mm LVDT which was fixed on both sides of the sample. And the sampling frequency was $1 \mathrm{~Hz}$. The sample and the LVDT were shown as the following figure.2a.

\section{Preparation of Production the Video in this Study}

The object video sequences Product is located in a laboratory at Wuhan University of Technology. The steps of producing the video for this study is as follows: firstly is to put the Specimen in Universal Testing Machine (Instron 5882) as shown in Figure 2.a . Second step is to apply automatic loading down as the same time reading the magnitude of applied loading in the programmer; then the next step is performed if the cracks start; by locating the digital microscope camera ( VHX-600E) as shown in Figure 2.b.

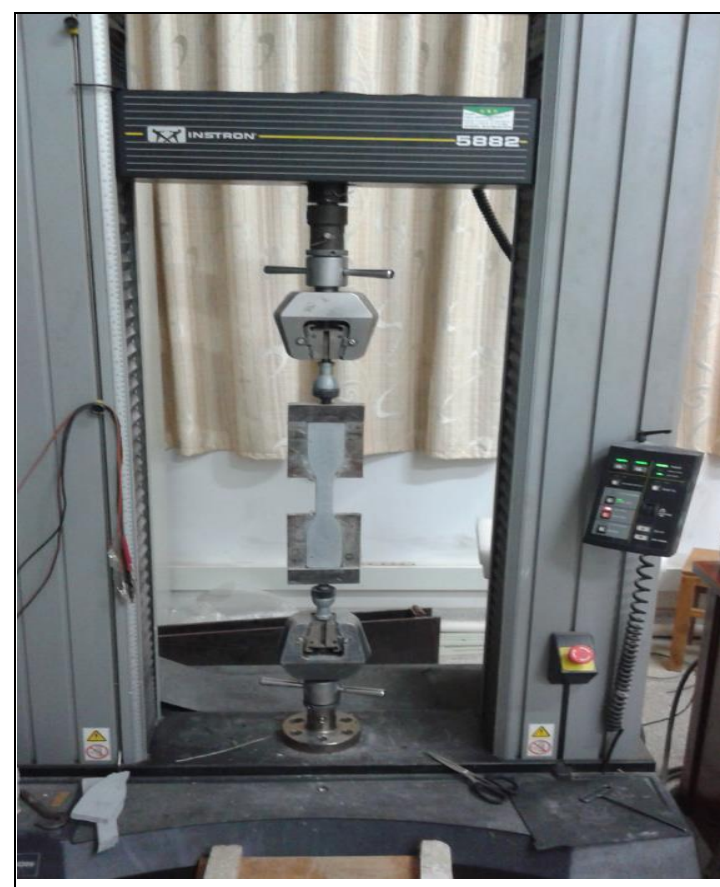

(a) The Universal Testing Machine (Instron 5882)

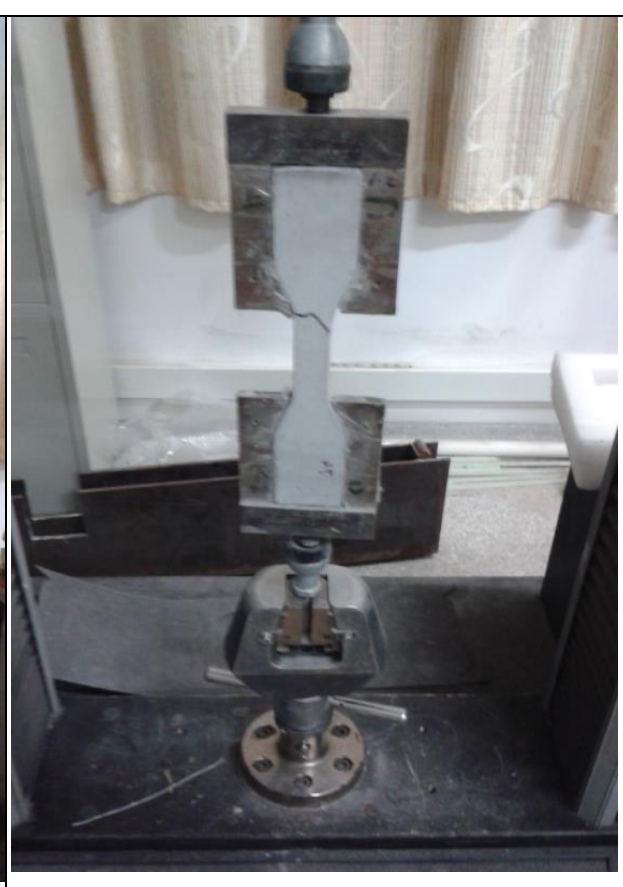

The Specimen of the video in this paper . 


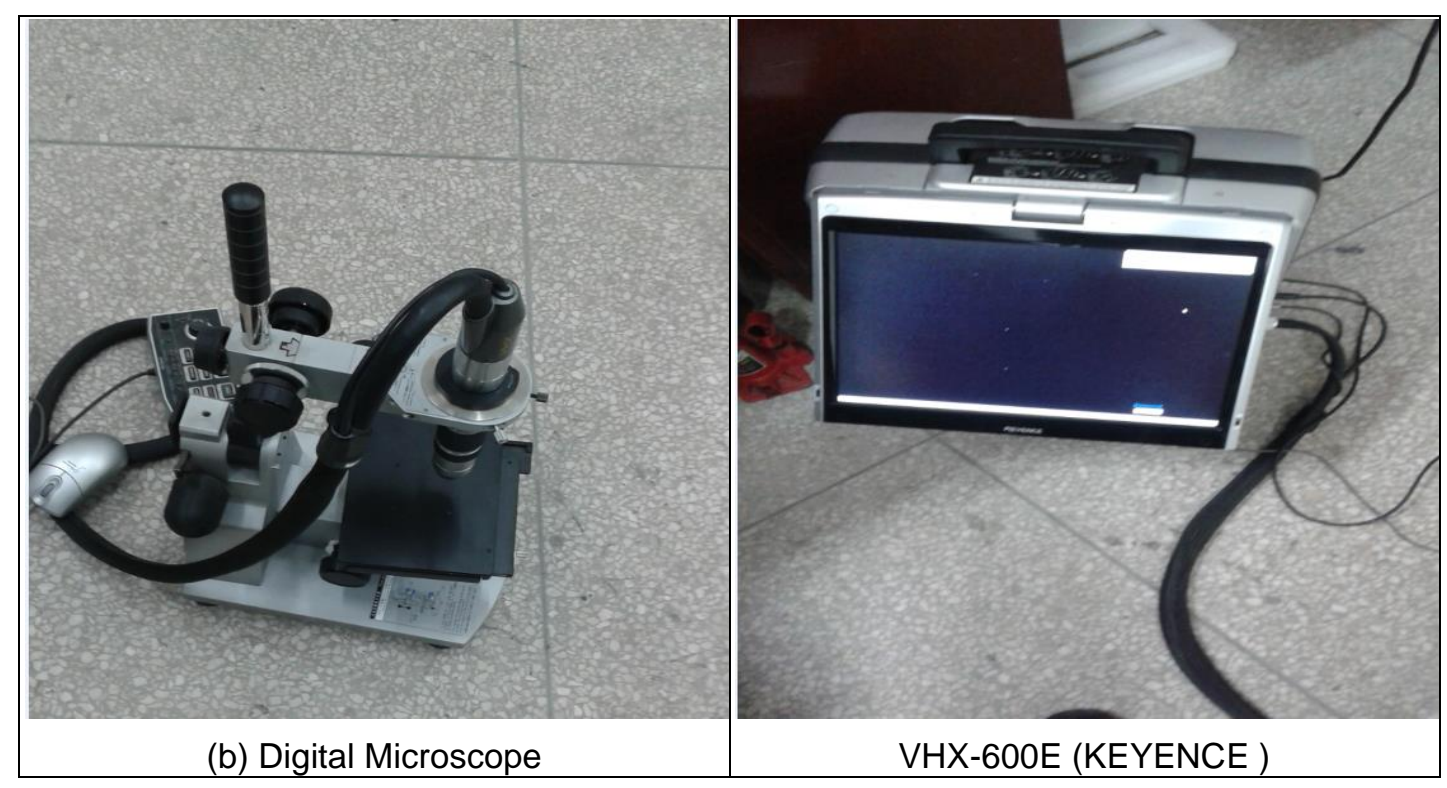

Figure 2. The Equipment of the Product the Video in this Paper

\section{Experimental and Discussion:}

\subsection{Experiment and Results}

In this section, we show experimental result of the proposed method. The proposed algorithm was implemented in MATLAB. (R2012 b).We experimentally test some result; we then compare our proposed method with three existing algorithms.

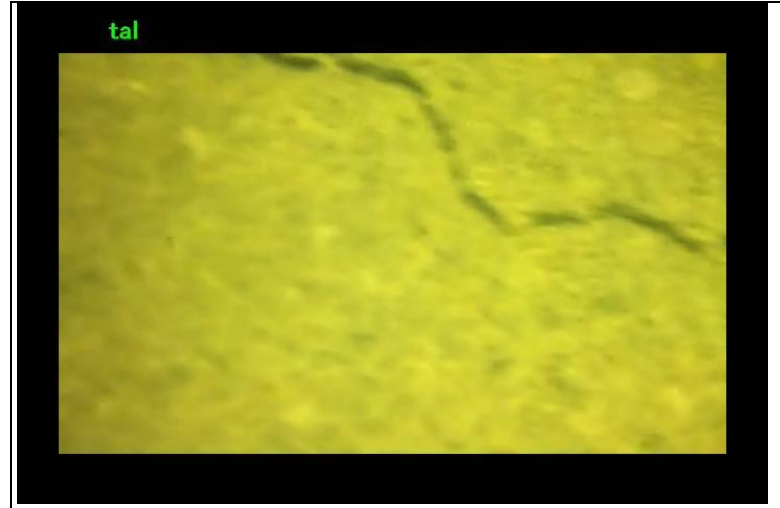

(a) Original Video

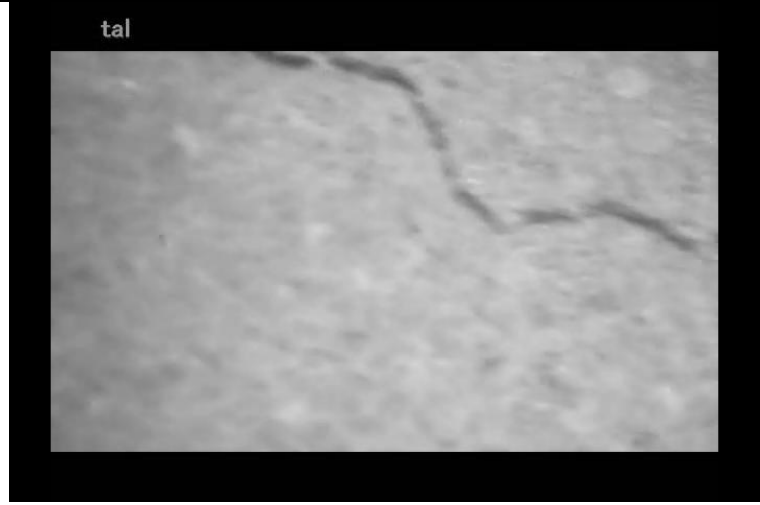

(b) Gray Image 


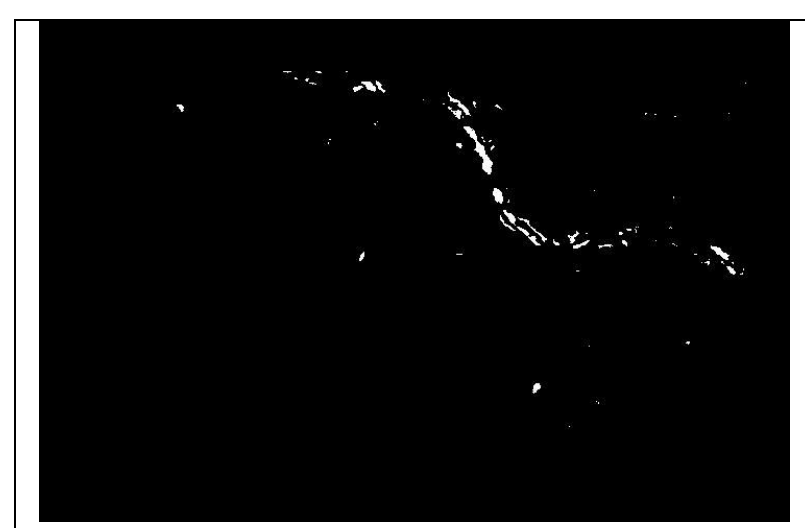

(c) Two difference image

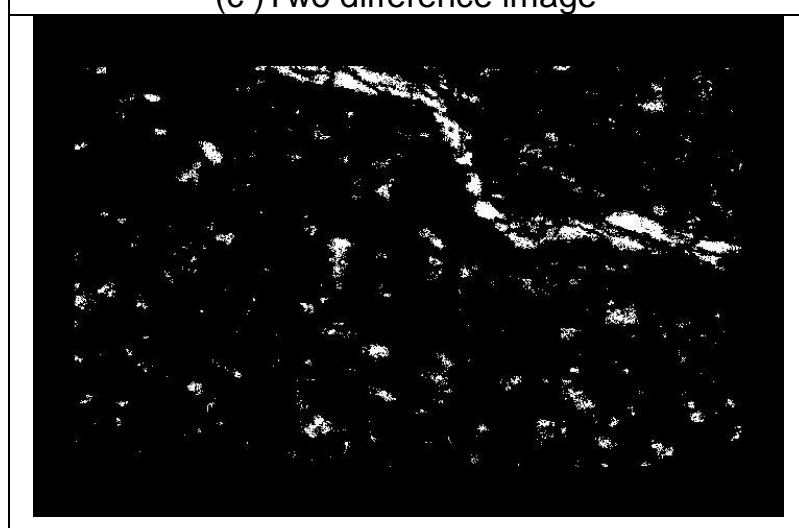

(e) GMM

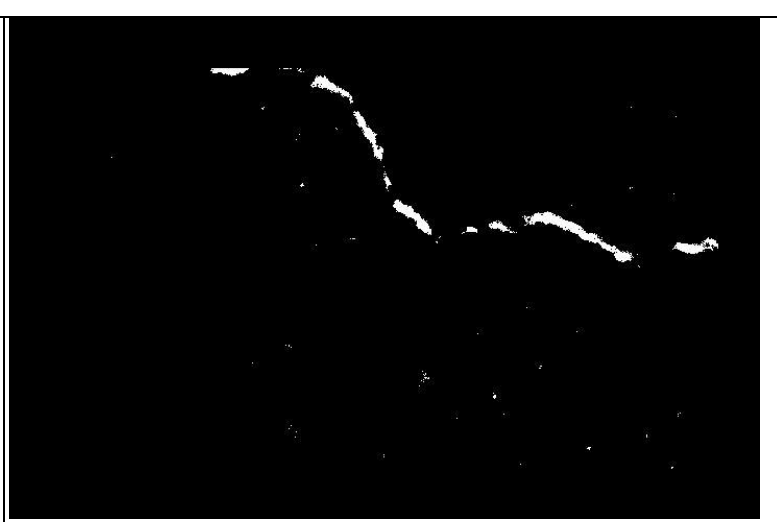

(d) Vibe Method

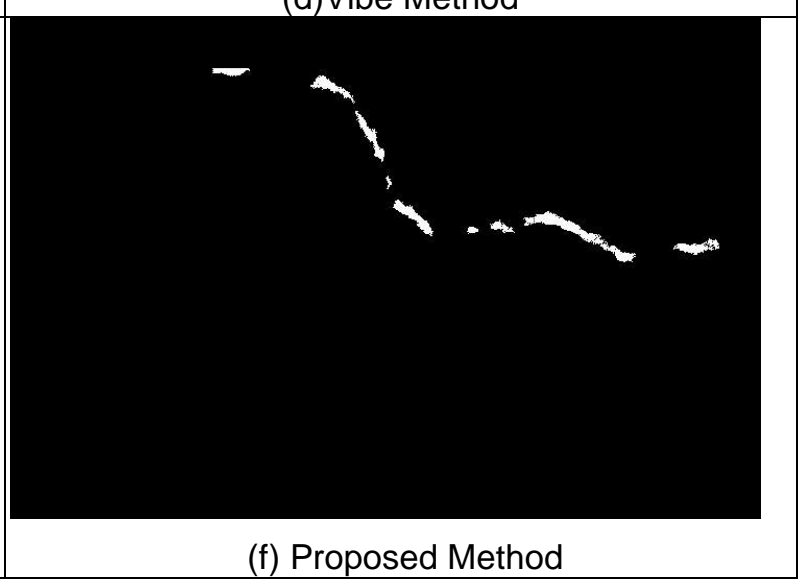

\section{Figure 3. Moving Crack (Frame 94) Comparative Foreground Segmentation Methods}

The object video sequences Product in laboratory in Wuhan University of Technology. The extracted moving crack in figure (3) by the proposed method is clearly when compared with the other methods. Moreover, the resulted image has no noise than the other approaches. The Crack in Two difference image is not clear and has noise and complete, while the resulted crack of the Vibe methods is clear and has noise. The GMM is find crack but has highest noise in contrast with the other methods.

\subsection{Quantitative Evaluations}

Quantitative evaluation of proposed method and comparison with Vibe Method. Methods were also performed in this study. The results were evaluated quantitatively From the comparison with the "ground truths" in terms of:

(1) The Number of False Positive (F): the number of background points that are misdetected as foreground.

(2) The Number of True Positives (T): the number of foreground points that are detected as foreground points[3]. 


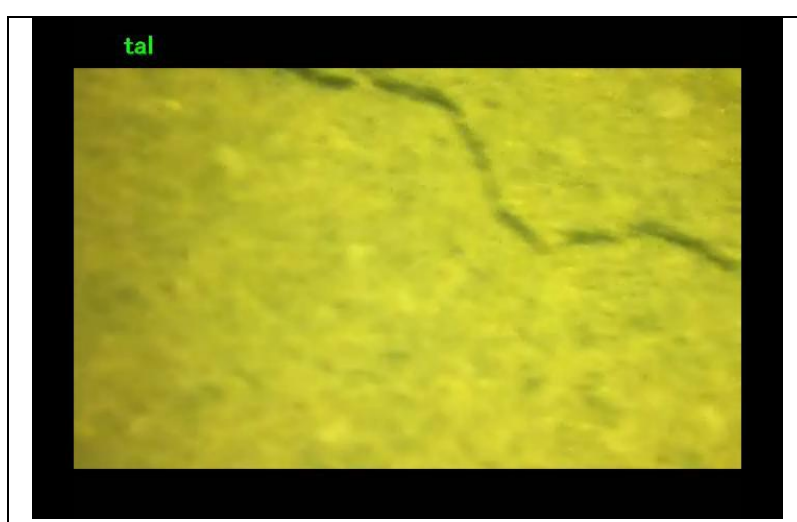

(a) Original Image

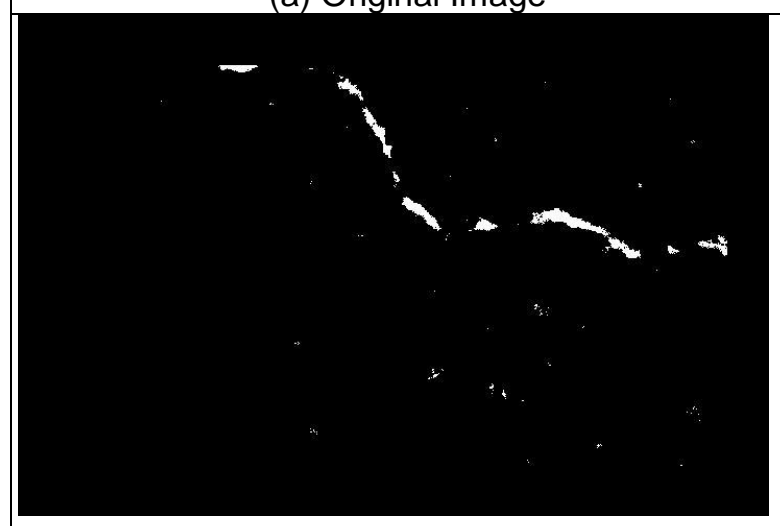

(c) Vibe Method

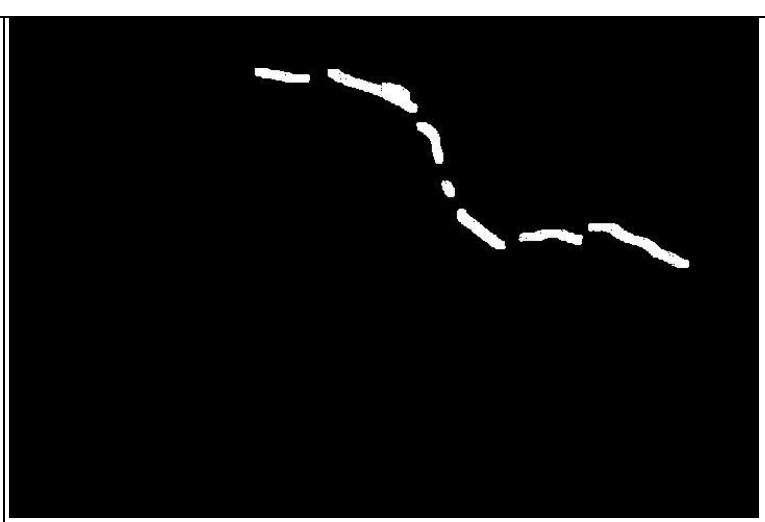

(b) Ground-truth

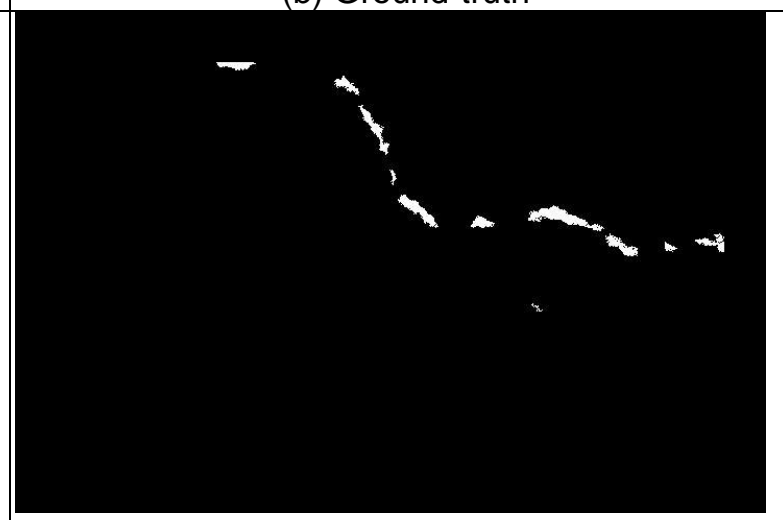

(d) Proposed Method

Figure.4. Moving Crack (Frame 93) Comparative Foreground Segmentation Methods

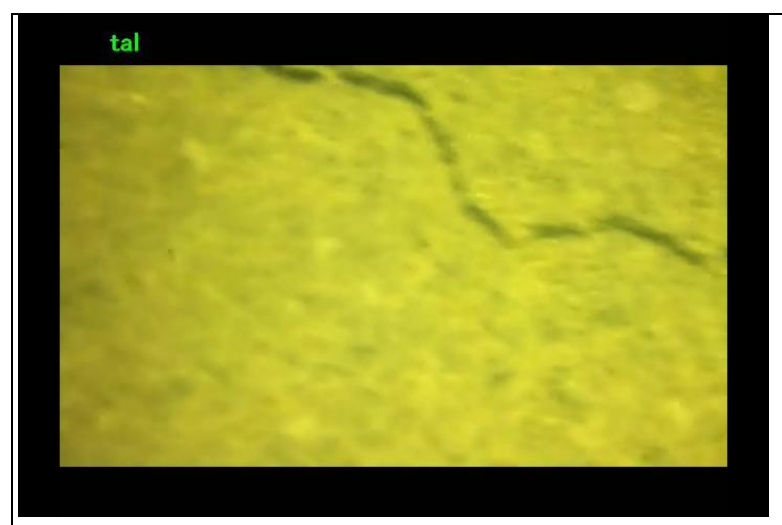

(a) Original Image

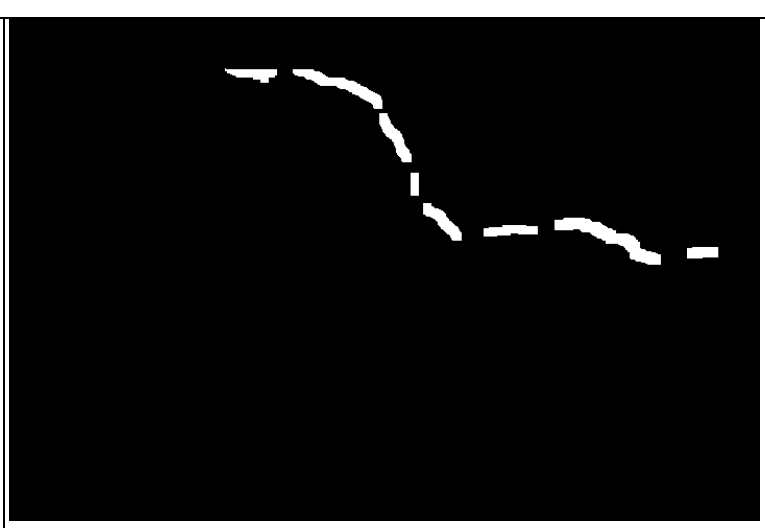

(b) Ground-truth 


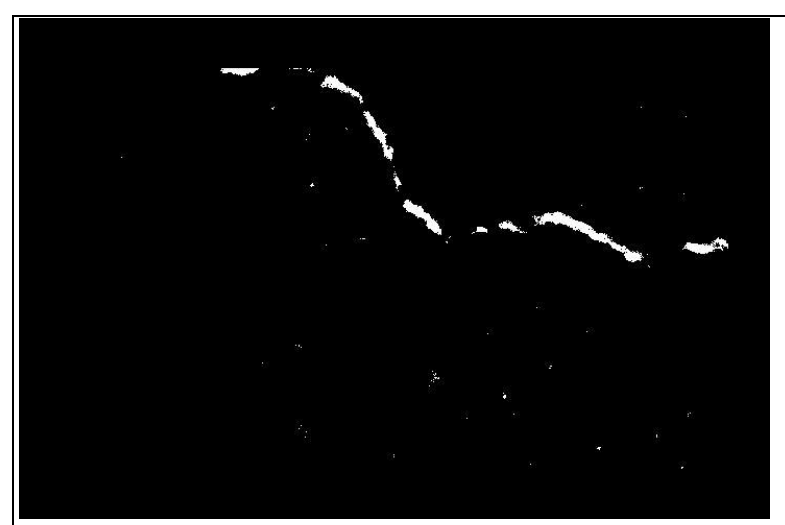

(c) Vibe Method

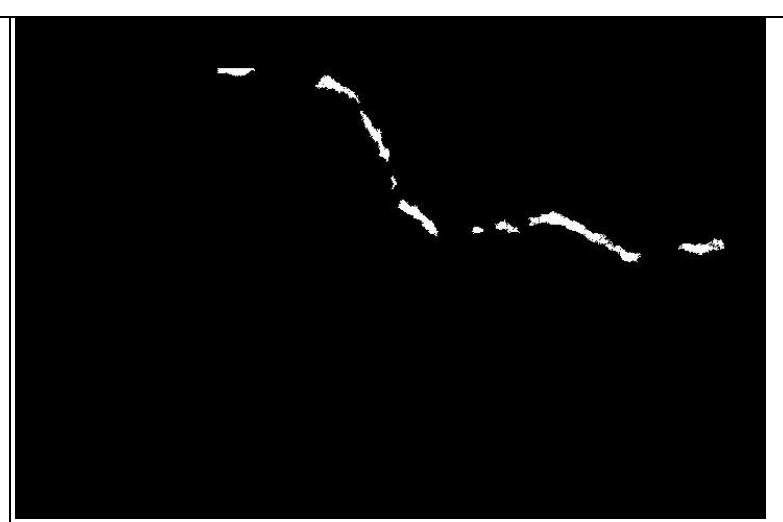

(d) Proposed Method

Figure 5. Moving Crack (Frame 94) Comparative foreground segmentation methods

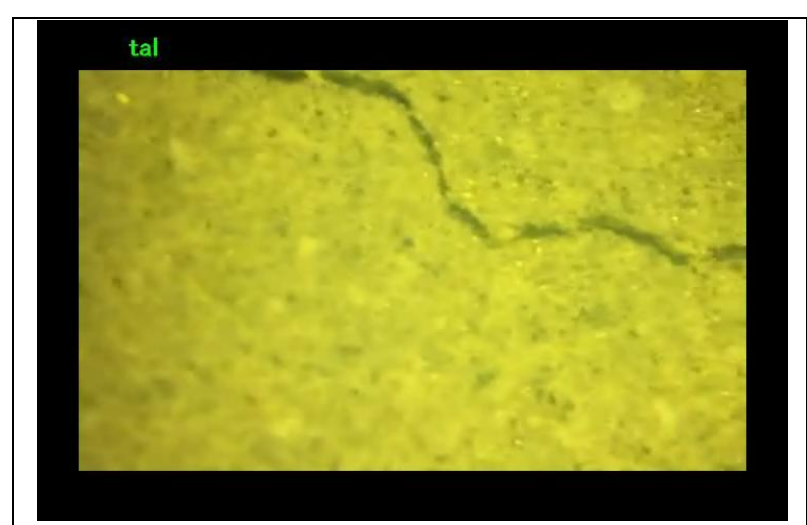

(a) Original Image

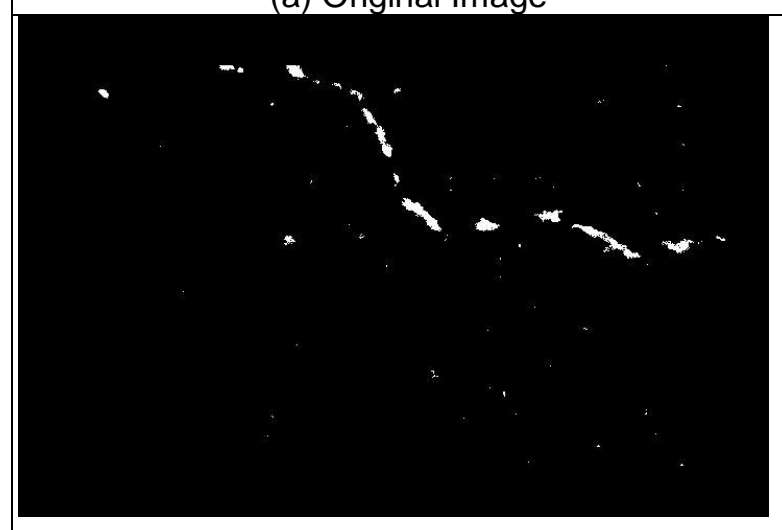

(c) Vibe Method

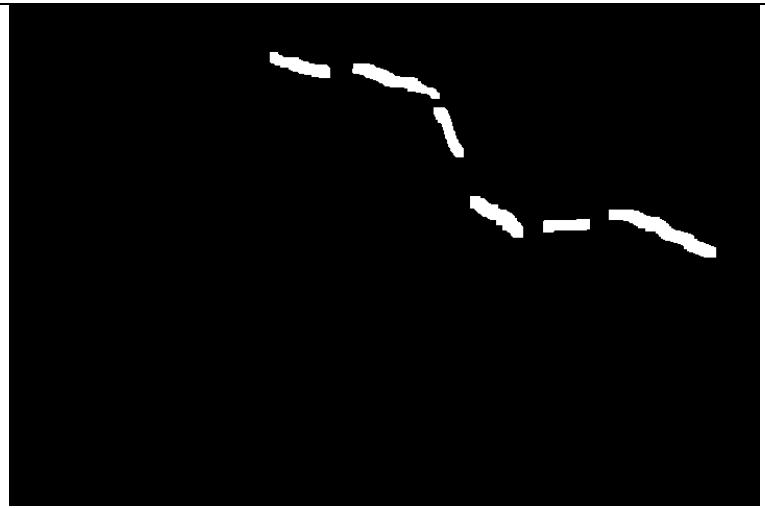

(b) Ground-truth

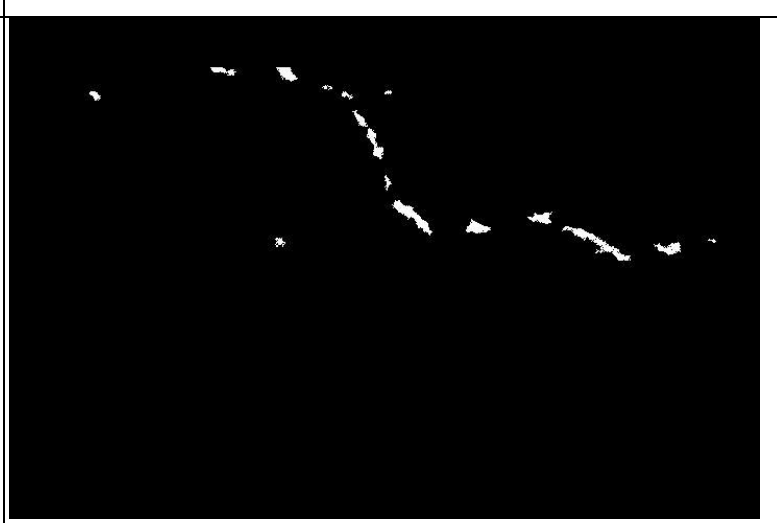

(d) Proposed Method

Figure 6. Moving Crack (Frame 100) Comparative Foreground Segmentation Methods 


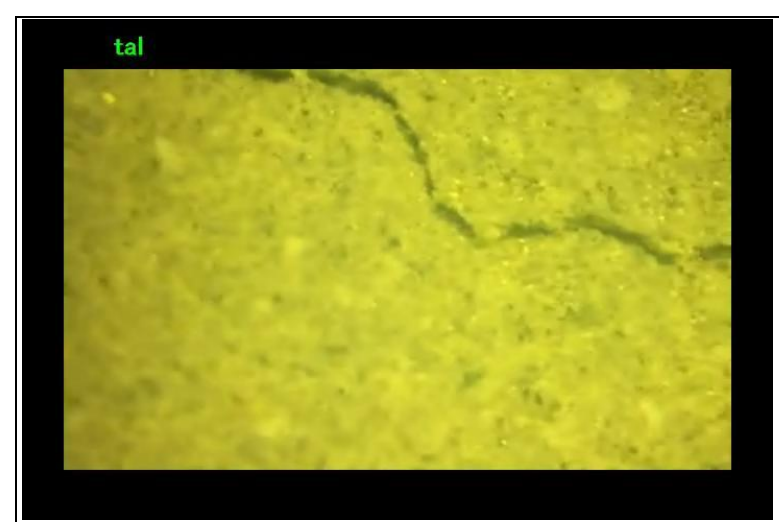

(a) Original Image

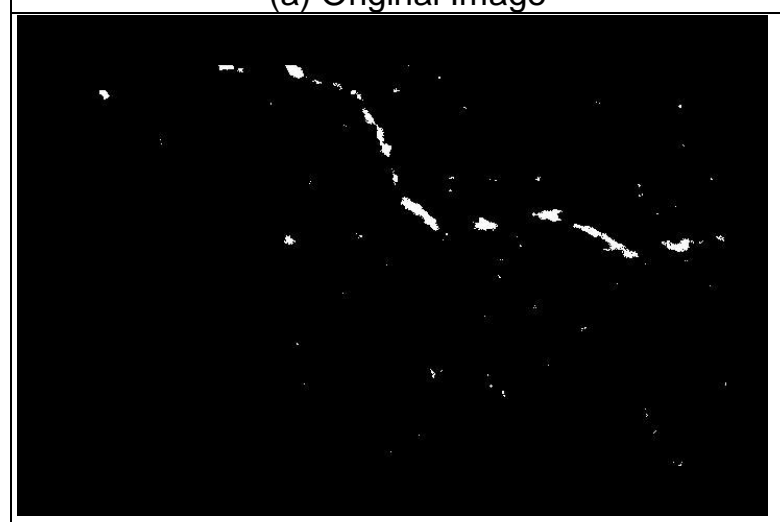

(c) Vibe Method

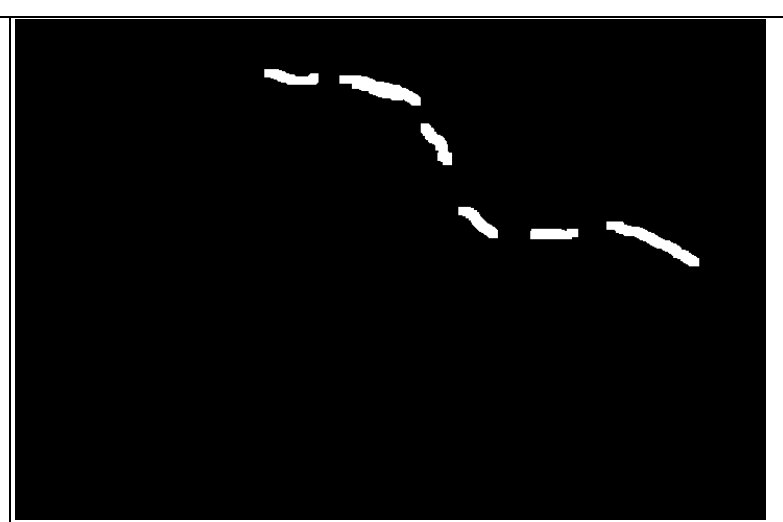

(b) Ground-truth

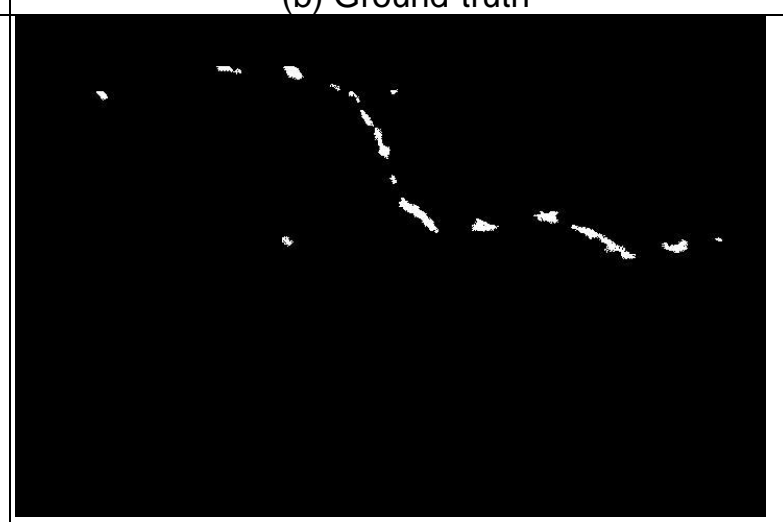

(d) Proposed Method

\section{Figure 7. Moving Crack (Frame 101) Comparative Foreground Segmentation Methods}

The extracted moving crack detection in figures (4-7) by the proposed method is clearly when compared with the Vibe method. Moreover, the resulted image has less noise than the Vibe Method. The Crack in our method is clearly and complete than Vibe method.

Table 2: Number of True Positives of Video Moving Crack Detection

\begin{tabular}{|c|c|c|}
\hline Method frames & Vibe Method & Proposed Method \\
\hline 93 & 43 & 48 \\
\hline 94 & 139 & 158 \\
\hline 100 & 113 & 114 \\
\hline 101 & 122 & 133 \\
\hline
\end{tabular}

The quantitative evaluation results over the examples displayed in Figs.(4-7) are shown in Tables 2 respectively. The quantitative evaluation agrees with the conclusions from the visual observation of the experimental results (Fig.8). 


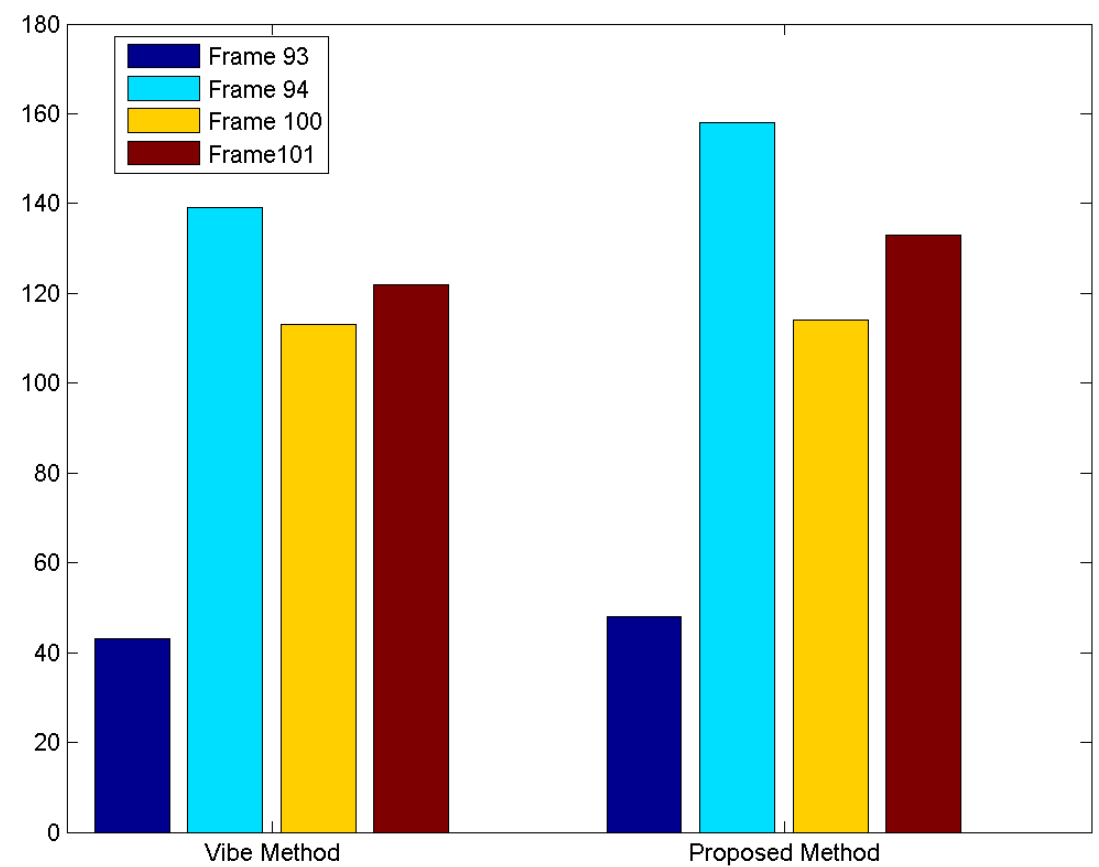

Figure 8. Number of True Positives of Moving Crack Detection four Frames

\section{Conclusion}

This paper propose a new approach in image processing method for detecting moving cracks in video. In this method, we use more than one filter like filter of Area and change the small area to background, and detecting major cracks using improved VIBE and Multiple Filtering in Image Processing Techniques. The experimental results of the proposed method in this paper successfully extract background and foreground image .The advantage of this method is clearly and accurate detection moving cracks in video. Also Experimental results show that the quantitative detection indicators of the proposed algorithm perform better than Vibe method.

\section{Acknowledgment}

The authors would like to thank all students in the laboratory in school of Science at Wuhan University of Technology to assist in the preparation of the specimen in this paper, and gratefully appreciate Mr. Xiong jiang for his assistance within the research.

\section{References}

[1] O. Barnich and M. V. Droogenbroeck, "VIBE: A powerful random technique to estimate the background in video sequences", University of Li'ege Montefiore Institute, INTELSIG Group Li'ege, ICASSP 2009, (2009) , pp. 945-948.

[2] M. Olivier Barnich and M.V. Droogenbroeck, "ViBe: A Universal Background Subtraction Algorithm for Video Sequences", IEEE Transactions on Image Processing, vol. 20, (2011), pp.1709-1724,.

[3] J. Dou and J. Li, "Moving object detection based on improved VIBE and graph cut optimization", OptikInternational Journal for Light and Electron Optics, vol. 124, (2013), pp. 6081-6088.

[4] M. Piccardi, "Background subtraction techniques: a review *," 2004 IEEE international conference on Systems, Man and Cybernetics, (2004), pp. 3099-3104.

[5] R. J. Radke, S. Andra, O. Al-Kofahi, and B. Roysam, "Image change detection algorithms: a systematic survey," IEEE Transactions on Image Processing, vol. 14, pp. 294-307, (2005).

[6] T. Bouwmans, "Recent advanced statistical background modeling for foreground detection-a systematic survey," Recent Patents on Computer Science, vol. 4, (2011) , pp. 147-176. 
[7] C. Stauffer and W. E. L. Grimson, "Learning patterns of activity using real-time tracking," IEEE Transactions on Pattern Analysis and Machine Intelligence, vol. 22, (2000), pp. 747-757.

[8] Y. Fan, "A real-time algorithm of dynamic background extraction in image sequence," in Proceedings of the Fourth International Conference on Machine Learning and Cybernetics, (2005); Guangzhou.

[9] S.-W. Chen, W. Luke K, and J.-H. Lan, "Moving Object tracking Based on Background Subtraction Combined Temporal Difference", presented at the International Conference on Emerging Trends in Computer and Image Processing (ICETCIP'2011), (2011); Bangkok.

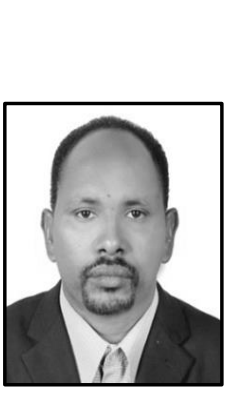

\begin{abstract}
Authors
Ahmed Mahgoub Ahmed Talab. Received his B.Sc degree in Mathematics of Sudan University of Science and Technology in 2002. $\mathrm{He}$ received his M.Sc degree in Industrial and Computational Mathematics in University of Khartoum in 2007. Currently, he is a Ph.D. candidate in Applied Mathematics at Wuhan University of Technology. His research interests include image processing, background subtraction, detection moving objects. Email : ahmedtalab10@yahoo.com.
\end{abstract}

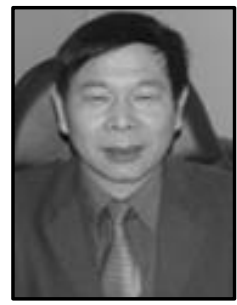

Zhangcan Huang. Received his B.Sc degree in Applied Mathematics of Jilin University in 1983. He received his PhD degree in Software and computer theory in Wuhan University in 2004. Currently he is a professor at School of Science in Wuhan University of Technology. His research interests include image processing and Intelligent computing.

Email: huangzc@whut.edu.cn.

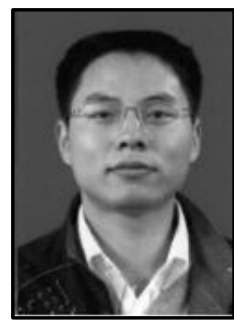

Fang xi. Received his B.Sc degree in Wuhan University of Technology. He received his M.Sc in Wuhan University of Technology. Currently he is Associate professor at School of Science in Wuhan University of Technology. His research interests include image processing and Intelligent computing.

Email: fangxi@whut.edu.cn.

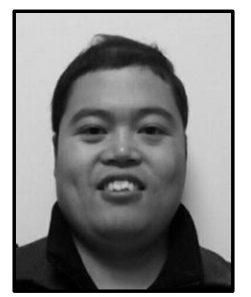

LiuHaiming. Received his B.Sc degree in information and computing science in Wuhan University of Technology. He received his M.Sc in applied mathematics in Wuhan University of Technology. His research interests include image processing and Intelligent computing.

Email : 1golden@163.com 